\title{
Characterization of Chemisorbed Hyaluronic Acid Directly Immobilized on Solid Substrates
}

\author{
Kahp Y. Suh, ${ }^{1,2}$ Jen Ming Yang, ${ }^{1,3}$ Ali Khademhosseini, ${ }^{4}$ David Berry, ${ }^{4,5}$ Thanh-Nga T. Tran, ${ }^{5}$ \\ Hyongshin Park, ${ }^{6}$ Robert Langer ${ }^{1,4,5}$ \\ ${ }^{1}$ Department of Chemical Engineering, Massachusetts Institute of Technology, Cambridge, Massachusetts 02139 \\ ${ }^{2}$ School of Mechanical and Aerospace Engineering, Seoul National University, Seoul 151-742, Korea \\ ${ }^{3}$ Department of Chemical and Materials Engineering, Chang Gung University, Kwei-Shan, Tao-Yuan 333, Republic of China
}

${ }^{4}$ Division of Biological Engineering, Massachusetts Institute of Technology, Cambridge, Massachusetts 02139

${ }^{5}$ Harvard-MIT Division of Health Sciences and Technology, Massachusetts Institute of Technology, Cambridge, Massachusetts 02139

${ }^{6}$ Center for Space Research, Massachusetts Institute of Technology, Cambridge, Massachusetts 02139

Received 12 February 2004; revised 8 June 2004; accepted 13 July 2004

Published online 14 October 2004 in Wiley InterScience (www.interscience.wiley.com). DOI: 10.1002/jbm.b.30152

\begin{abstract}
Hyaluronic acid (HA) has a number of potential biomedical applications in drug delivery and tissue engineering. For these applications, a prerequisite is to understand the characteristic of HA films directly immobilized to solid substrates. Here, we demonstrate that high molecular weight HA can be directly immobilized onto hydrophilic substrates without any chemical manipulation, allowing for the formation of an ultrathin chemisorbed layer. Hyaluronic acid is stabilized on these surfaces through hydrogen bonding between the hydrophilic moieties in HA [such as carboxylic acid (- COOH) or hydroxyl (-OH) groups] with silanol (- $\mathrm{SiOH}$ ), carboxylic acid or hydroxyl groups on the hydrophilic substrates. Despite the water solubility, the chemisorbed HA layer remained stable on glass or silicon oxide substrates for at least 7 days in phosphate-buffered saline. Furthermore, HA immobilized on silicon and other dioxide surfaces in much higher quantities than other polysaccharides including dextran sulfate, heparin, heparin sulfate, chondroitin sulfate, dermatan sulfate, and alginic acid. This behavior is related to the molecular entanglement and intrinsic stiffness of $\mathrm{HA}$ as a result of strong internal and external hydrogen bonding as well as high molecular weight. These results demonstrate that $\mathrm{HA}$ can be used to coat surfaces through direct immobilization. (C) 2004 Wiley Periodicals, Inc. J Biomed Mater Res Part B: Appl Biomater 72B: 292-298, 2005
\end{abstract}

Keywords: hyaluronic acid; direct immobilization; characterization; chemisorption; substrates

\section{INTRODUCTION}

Recently, polysaccharide coatings have attracted much attention in biomaterials research due to their ability to reduce fouling of surfaces by biological species. ${ }^{1-11}$ Although the

\footnotetext{
Correspondence to: R. Langer (e-mail: rlanger@mit.edu or sky4u@snu.ac.kr) Contract grant sponsor: U.S. Army Institute for Soldier Nanotechnologies1; contract grant number: DAAD-19-02-D0002

Contract grant sponsor: Brain Korea 21 Project

Contract grant sponsor: National Science Council of Republic of China; contract grant number: NSC-40212-F

Contract grant sponsor: Chang Gung Hospital

Contract grant sponsor: Howard Hughes Medical Institute

(ㄷ) 2004 Wiley Periodicals, Inc.
}

mechanism of protein or cell resistance is not completely understood, conformation and dehydration of polysaccharides through interactions with water, as well as charge interactions are believed to be important. ${ }^{1}$ A variety of polysaccharides have been explored for use as potential lowfouling surface modifiers. These include dextrans, ${ }^{2-5}$ carboxymethylated dextrans, ${ }^{6-8}$ and other carboxylated polysaccharides such as alginic acid and hyaluronic acid (HA). ${ }^{9-11}$ Of these polysaccharides, HA has received much attention due to its unique properties. Hyaluronic acid is a linear polysaccharide composed of repeating disaccharide units of $\mathrm{N}$-acetyl-D-glucosamine linked to D-glucuronic acid, and unlike other glycosaminoglycans (GAGs), HA is not 
sulfated. As a component of the extracellular matrix, HA plays an important role in lubrication, water sorption, water retention, and a number of cellular functions such as attachment, migration, and proliferation. ${ }^{12,13}$ Hyaluronic acid is therefore an attractive building block for new biocompatible and biodegradable polymers that have applications in drug delivery, tissue engineering, and viscosupplementation. ${ }^{14-17}$

The formation of a stable HA coating has potential biomedical applications ranging from bioactive surfaces to the formation of multilayer polyelectrolyte films. ${ }^{18-20}$ To generate HA-coated surfaces, various immobilization techniques have been employed ranging from covalent attachment, ${ }^{9,21-23}$ layer-by-layer deposition, ${ }^{24,25}$ and binding with natural ligands such as $\mathrm{p} 32 .{ }^{26}$ These strategies, however, involve potentially complicated synthetic approaches that require the use of chemicals, UV light, or cumbersome procedures to prepare additional binding layers, limiting their potential as a general route to HA surface immobilization.

Here, we demonstrate the formation of a stable, chemisorbed HA layer on hydrophilic surfaces, such as glass and silicon oxides, and characterize it using X-ray photoelectron microscopy (XPS), ellipsometry, and atomic force microscopy (AFM). In addition, we examined the underlying mechanism by studying the HA layer formation at various $\mathrm{pH}$ conditions and with washing procedures. Evidence suggests that the HA is stabilized on the surface through hydrogen bonding between the hydrophilic moieties in HA, such as carboxylic acid $(-\mathrm{COOH})$ or hydroxyl $(-\mathrm{OH})$ groups with silanol (- $\mathrm{SiOH})$, carboxylic acid or hydroxyl groups on the hydrophilic substrates. The chemisorbed HA layer remains stable in phosphate-buffered saline (PBS) for at least 7 days without losing its resistant properties. HA is therefore an important biological molecule that can be directly immobilized on substrates with high efficiency and stability.

\section{MATERIALS AND METHODS}

\section{Materials}

HA (lot no. $904572, M_{\mathrm{n}}=2.1 \mathrm{MDa}$ by light scattering) was kindly supplied by Genzyme Inc. (Boston, MA). Silicon dioxide wafers $\left(1 \mu \mathrm{m}\right.$ of $\mathrm{SiO}_{2}$ on $\left.\mathrm{Si}\right)$ were purchased from International Wafer Service (Portola Valley, CA) and used without further treatment. Heparin and heparin sulfate (HS) were from Celsus Laboratories (Columbus, $\mathrm{OH}$ ). Chondroitin sulfate (CS) A, CS C, dermatan sulfate (DS), fluorescein isothiocyanate-labeled bovine serum albumin (FITC-BSA), goat anti-rabbit immunoglobulin G (FITC-IgG), fibronectin (FN), and anti-FN antibody were purchased from Sigma (St. Louis, MO). Glass slides were treated with $\mathrm{O}_{2}$ plasma for 1 min to generate $-\mathrm{OH}$ groups as well as to clean the surfaces unless otherwise indicated.

\section{Surface Characterization}

Fluorescent optical images were obtained using an inverted microscope (Axiovert 200, Zeiss). X-ray photoelectron spectroscopy spectra were recorded using a Kratos AXIS Ultra spectrometer. Spectra were obtained with a monochromatic $\mathrm{Al} \mathrm{K} \mathrm{K}_{\alpha} \mathrm{X}$-ray source $(1486.6 \mathrm{eV})$. Pass energy was 160 $\mathrm{eV}$ for survey spectra and $10 \mathrm{eV}$ for high-resolution spectra. All spectra were calibrated with reference to the unfunctionalized aliphatic carbon at a binding energy of $285.0 \mathrm{eV}$. Spectra were recorded with similar settings (number of sweeps, integration times, etc.) from sample to sample to enable comparisons to be made. The analysis of the XPS spectra was performed on the basis of $90^{\circ}$ unless otherwise indicated. Atomic force micrographs were obtained with tapping mode on a NanoScope III Dimension (Veeco Instruments, Rochester, NY) in air. The scan rate was $0.5 \mathrm{~Hz}$ and 256 lines were scanned per sample. Tapping mode tips, NSC15 - $300 \mathrm{kHz}$, were obtained from MikroMasch (Portland, OR). Data were processed using Nanoscope III 4.31r6 software (Veeco Instruments Inc.). The thickness of the chemisorbed HA layer was measured with a Gaertner L116A ellipsometer (Gaertner Scientific Corp., Skokie, IL) with a $632.8 \mathrm{~nm} \mathrm{He}-\mathrm{Ne}$ laser. A refractive index of 1.46 was used for all HA films, and a three-phase model was used to calculate thicknesses.

\section{Construction and Stability of a Chemisorbed Layer and Testing Protein Adsorption}

A few drops of HA solution $(5 \mathrm{mg} / \mathrm{mL}$ in distilled water) were placed on the surface and spin coated (Model CB 15, Headaway Research, Inc.) at $1000 \mathrm{rpm}$ for $10 \mathrm{~s}$. The samples were stored overnight at room temperature to allow the solvent to evaporation. To examine the effect of washing, some samples were washed several times within 30 min of spin coating and then dried with a mild nitrogen stream. To examine the effect of $\mathrm{pH}$, the silicon oxide surfaces were exposed for several hours to the solutions of $\mathrm{pH} 2,7$, and 11, respectively, leading to different oxidization states. HA films were prepared on those surfaces using the same procedure described above. In addition to HA, thin films of the other polysaccharides were prepared in the same manner.

To measure the immobilization of HA, heparin, HS, CS A, CS C, and DS films, we performed fluorescent staining for adhesion of various proteins on the coated surfaces. FITCBSA $(50 \mu \mathrm{g} / \mathrm{mL}), \operatorname{IgG}(50 \mu \mathrm{g} / \mathrm{mL})$, and FN $(20 \mu \mathrm{g} / \mathrm{mL})$ were dissolved in PBS solution ( $\mathrm{pH}=7.4 ; 10 \mathrm{~m} M$ sodium phosphate buffer, $2.7 \mathrm{~m} M \mathrm{KCl}$, and $137 \mathrm{~m} M \mathrm{NaCl}$ ). To measure FN adsorption, the surfaces were stained with anti-FN antibody for $45 \mathrm{~min}$, followed by a $1 \mathrm{~h}$ incubation with the FITC-IgG antibody. A few drops of the protein solution were evenly distributed onto the HA surfaces. After storing at room temperature for $30 \mathrm{~min}$, the surfaces were rinsed with PBS solution and water and then blown dry in a stream of nitrogen. To analyze stability, HA surfaces were placed in a PBS bath at various times and stored at room temperature for up to 7 days. The PBS solution was changed daily to prevent readsorption of dissociated HA onto the surface. The stability was subsequently analyzed by testing for FN adsorption. The slides were then examined under a fluorescent microscope 

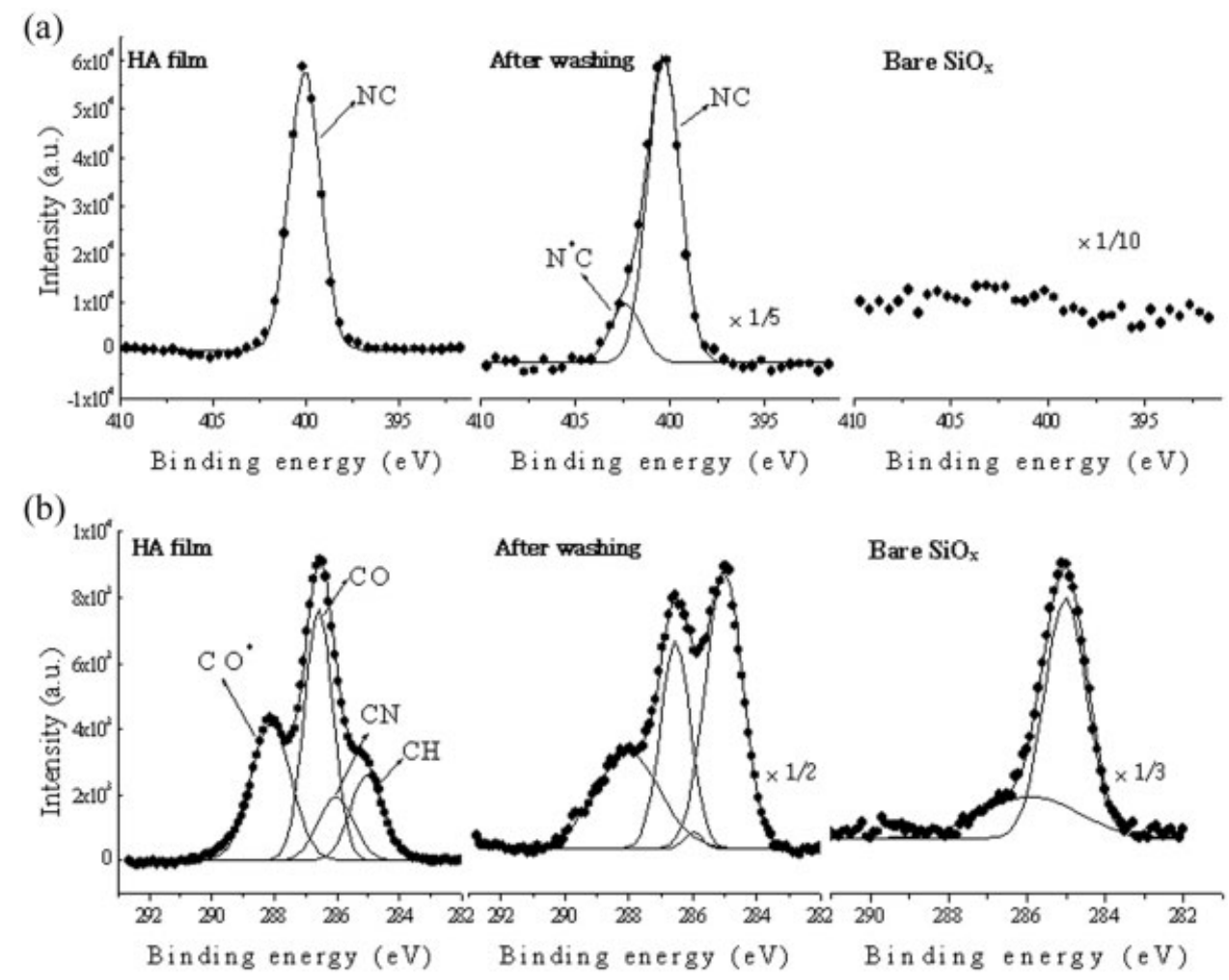

Figure 1. High-resolution XPS spectra for (a) nitrogen (N 1s) and (b) carbon (C 1s) peaks in HA recorded for as-spun, washed, and bare silicon oxide substrates. For carbon peaks of an as-spun and a washed film, the spectra were deconvoluted with four Gaussian peaks that are assigned at each oxidized state. For convenience, the peak for the strongly oxidized carbon (CO*) was not deconvoluted in detail. All films were prepared and characterized on the silicon oxide substrate to take advantage of the flat surface.

under a UV light exposure of $2 \mathrm{~s}$. Blank glass slides with or without FN staining were used as positive and negative controls respectively. Fluorescent images were analyzed quantitatively using Scion Image and the statistical analysis was performed using one-sided ANOVA tests with $p<0.05$ to distinguish between statistical significance.

\section{RESULTS AND DISCUSSION}

\section{Detection of a Chemisorbed HA Layer}

The presence of a chemisorbed HA layer on silicon dioxide substrates or glass was verified by analyzing the elemental composition (carbon, oxygen, nitrogen, and silicon) of the surfaces using XPS. In particular, the detection of nitrogen in the XPS spectra was strong evidence to support the presence of a residual HA layer (Figure 1) because nitrogen is found in HA but not the substrate. As expected, no nitrogen was detected on the bare silicon oxide. The intensity at $400.1 \mathrm{eV}$ $(\mathrm{N} 1 \mathrm{~s})$ decreased to about $25 \%$ of its original intensity after washing with PBS, though the peak remained, indicating a residual layer of HA [Figure 1(a)]. A new XPS peak was also detected at $402.3 \mathrm{eV}(15.5 \%)$ after washing, suggesting a modified oxidation state of nitrogen, denoted $\mathrm{N}^{*} \mathrm{C}$. We hy- pothesize that the new peak originates from the partial protonation or hydrogen bonding of nitrogen to silanol groups (- $\mathrm{SiOH})$ on the surface. The persistence of the nitrogen peak and the emergence of a new oxidized state $\left(\mathrm{N}^{*} \mathrm{C}\right)$ generated after washing are consistent with a residual layer on the surface formed by chemical interactions between the layer and the substrate.

The carbon peak (C 1s) of an as-spun film contains four peaks that are located at 285.0 (16.1\%), $286.1(12.7 \%), 286.6$ (40.0\%), and 288.1 (31.2\%), consistent with previous reports [Figure 1(b)]. ${ }^{27}$ The amount of unfunctionalized hydrocarbon $(285 \mathrm{eV})$ was higher than expected $(7.1 \%),{ }^{27}$ which may be attributed to carbon adsorption from the air. In order of increasing binding energies these peaks represent the hydrocarbon environment $(\mathrm{HC})$, carbon singly bound to nitrogen $(\mathrm{CN})$, carbon singly bound to oxygen $(\mathrm{CO})$, strongly oxidized carbons $(\mathrm{CO} *)$ including carbon doubly bound to oxygen and a combined peak representing both amide and carboxylate ion carbon atoms (CON and COO). ${ }^{27}$ In contrast to the as-spun coatings, the relative intensities were substantially changed after washing with the peak locations slightly shifted. Two factors potentially responsible for this behavior are the increased portion of unfunctionalized hydrocarbon from the substrate, and the surface interactions between HA and the 


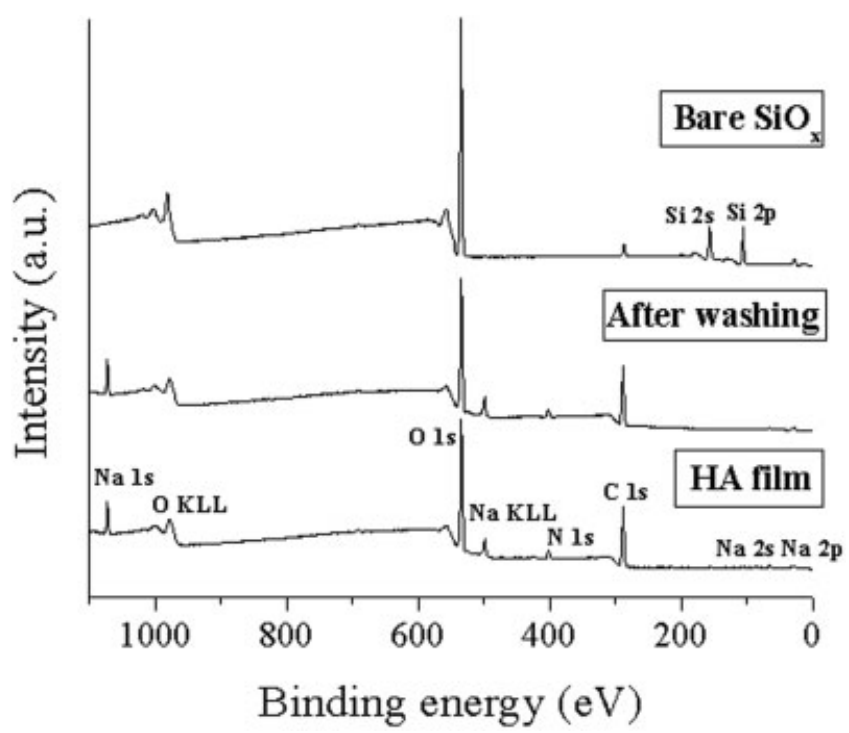

Figure 2. The wide scans of XPS spectra for as-spun, washed, and bare silicon oxide substrates. The results indicate that the substrate surface is nearly fully covered with the chemisorbed layer.

substrate. Based on the modified oxidation state of nitrogen in the XPS spectra and hydrophilic moieties in HA, some strong interactions, such as hydrogen bonding, may play an important role in the formation of the chemisorbed layer. In a separate experiment, the HA film was completely washed away on hydrophobic substrates such as untreated polystyrene (data not shown), which indicates that other hydrophobic interactions could be ruled out in examining the origin of the chemisorbed layer.

To analyze the thickness of the HA film, we used ellipsometry, AFM, and XPS measurements at two different an- gles. At a $90^{\circ}$ take-off angle (long penetration depth), silicon peaks were not seen for an as-spun sample (i.e., thick HA film on a glass), as opposed to bare silicon oxide and washed film controls. On the other hand, silicon peaks were nearly absent on the washed film when a $30^{\circ}$ take-off angle was used (short penetration depth; Figure 2). This indicates that the residual film was extremely thin, less than $5-10 \mathrm{~nm}$ depending on the element and electron selected, the thickness ranging within the penetration depth, and the substrate surface is nearly fully covered with the chemisorbed layer. The presence of the chemisorbed layer was further confirmed by ellipsometry and AFM measurements. The ellipsometry results indicated the initial thickness of the HA film was about $330 \mathrm{~nm}$, which decreased drastically to about $3 \mathrm{~nm}$ after washing and then remained at the same value. Furthermore, the roughness of a residual layer $(2.1 \mathrm{~nm})$ is between that of the substrate $(1.8$ $\mathrm{nm})$ and the as-spun film $(2.3 \mathrm{~nm})$, which also supports the presence of a residual layer (Figure 3 ).

To further explore the potential mechanism of adhesion, we exposed silicon oxide surfaces to three different $\mathrm{pH}$ values of 2,7 , and 11 to test the effects of surface charge and hydrophobicity on the formation of a HA coating (see the experimental protocol). At acidic conditions $(\mathrm{pH}=2)$, the hydroxyl groups present on the surface are protonated $\left(\mathrm{OH}_{2}^{+}\right)$ such that the adsorption of HA should be enhanced due to negative charge of HA. In contrast, because the surface is negatively charged $\left(\mathrm{O}^{-}\right)$, the adsorption would be reduced at basic conditions $(\mathrm{pH}=11)$. At $\mathrm{pH} 11$, the atomic mass percentage of nitrogen on the surface was $0.33 \%$ whereas it increased to $3.61 \%$ when exposed to $\mathrm{pH} 2$ (Table I). These results indicate that HA is more likely to adsorb to positively charged surfaces than negatively charged surfaces. Interestingly, neutral surfaces $(\mathrm{pH}=7)$ were also effective in ad- (a)
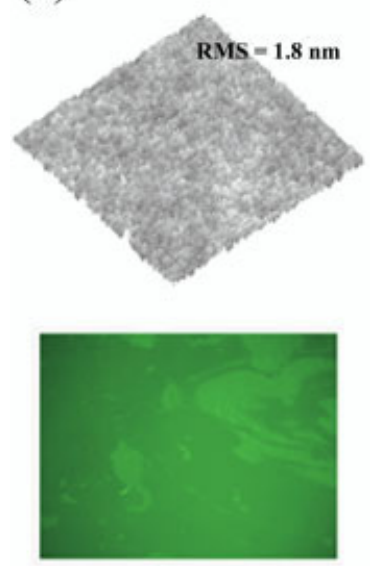

Bare $\mathrm{SiO}_{2}$ (b)
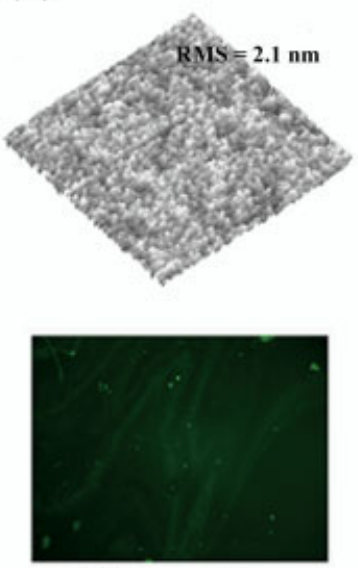

After washing (c)
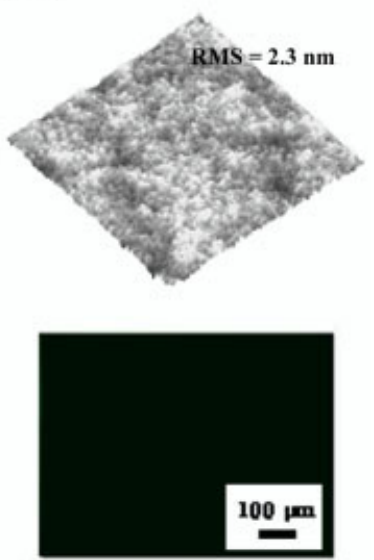

Before washing

Figure 3. Atomic force microscopy images of surface roughness and the corresponding fluorescent images for FN adsorption for (a) a bare silicon oxide substrate, (b) a HA surface after thorough washing, and (c) an as-coated HA film. Note that the roughness of the film after washing locates between those of the other two surfaces, supporting the presence of a chemisorbed layer. The height scale is $5 \mathrm{~nm}$ and the scan size is $1 \times 1 \mu \mathrm{m}^{2}$. The fluorescent images reveal that the surface is fully covered with HA even after extensive washing. 
TABLE I. Atomic Mass Percentage of Carbon, Nitrogen, Oxygen, and Silicon Elements for HA Films Formed under Various Conditions

\begin{tabular}{lcllr}
\hline & \multicolumn{4}{c}{ Atomic Conc. \% } \\
\cline { 2 - 5 } \multicolumn{1}{c}{ Sample } & $\mathrm{C}$ & $\mathrm{N}$ & $\mathrm{O}$ & $\mathrm{Si}$ \\
\hline Exposure to pH 2 & 57.6 & 3.6 & 34.0 & 4.8 \\
Exposure to pH 7 & 52.2 & 3.3 & 38.3 & 6.2 \\
Exposure to pH 11 & 14.6 & 0.3 & 58.0 & 27.1 \\
No washing + drying & 49.2 & 2.7 & 38.8 & 9.3 \\
Washing after 30 min & & & & \\
$\quad+$ drying & 11.7 & 0.7 & 64.6 & 23.0 \\
Bare silicon dioxide & 4.2 & 0 & 65.4 & 30.4 \\
\hline
\end{tabular}

Errors are within $5 \%$

hering HA (3.34\%), which also supports the presence of hydrogen bonding between HA and the hydroxyl groups.

We next explored whether the current approach is ubiquitous in immobilizing polymers having hydrophilic moieties on hydrophilic substrates. A previous study reported that carboxyl (- $-\mathrm{COOH})$ groups were confined onto hydrophilic surfaces with additional thermal polymerization. ${ }^{28}$ Poly(ethylene glycol)s, however, detach from the substrates upon hydration despite having hydrophilic moieties $(-\mathrm{OH})$. We hypothesize that two factors are responsible for the formation of a chemisorbed HA layer. First, hydrogen bonding should be strong enough to endure the polymer swelling stress at the interface upon exposure to water. Second, the chemisorbed layer should have a dense molecular structure such as entanglement to prevent penetration of water molecules. Thus, sufficiently strong hydrogen bonding is required to prevent the adsorbed layer from peeling off from the surface. In this regard, the HA film should have enough contact time with the surface to build a robust interface. As indicated by XPS, the amount of nitrogen adsorbed onto the surface was lower when the sample was washed within $30 \mathrm{~min}$ after spin coating $(0.69 \%$; Table I) and significantly increased to $2.74 \%$ when the sample was dried overnight prior to washing. This indicates that the duration of exposure and sample drying may be important in the adsorption of the HA onto the surfaces.

With respect to the density of the molecular structure, HA is a highly hydrated polyanion, which forms network between domains in solutions. ${ }^{29,30}$ In addition, the polymer shows intrinsic stiffness due to hydrogen bonds between adjacent saccharides. HA immobilized on silicon and other dioxide surfaces in much higher quantities than other polysaccharides including dextran sulfate, heparin, HS, CS, DS, and alginic acid (Table II) based on the highest nitrogen composition $(3.75 \%)$ and the lowest oxygen-to-carbon ratio $(0.64 \%)$. This behavior could be attributed to either differences between the molecular structures of various polysaccharides or their lower molecular weights compared to HA.

\section{Protein Resistance, Degradability, and Stability of a Chemisorbed HA Layer}

To test the effectiveness of the HA surfaces for protein resistance, HA-modified surfaces were exposed to FITC-
TABLE II. Atomic Mass Percentage of GAG Surfaces and Control Surfaces

\begin{tabular}{lllrr}
\hline Sample & N & O & \multicolumn{1}{c}{ C } & O:C \\
\hline Untreated & 0.00 & 92.4 & 7.6 & 12.2 \\
HA & 3.8 & 37.5 & 58.7 & 0.6 \\
Heparin & 0.2 & 89.4 & 10.4 & 8.6 \\
HS & 0.1 & 91.1 & 8.8 & 10.4 \\
CS A & 0.5 & 88.8 & 10.7 & 8.3 \\
CS C & 0.1 & 90.5 & 9.4 & 9.6 \\
DS & 0.4 & 89.0 & 10.6 & 8.4 \\
\hline
\end{tabular}

XPS was performed on GAG surfaces formed on silicon dioxide after washing. Untreated surfaces are silicon dioxide only. Numbers for nitrogen, oxygen, and carbon refer to atomic mass percentage. Oxygen:Carbon is the atomic mass percentage of oxygen divided that by carbon. Errors are within $5 \%$.

BSA, FITC-IgG, and FN. The adhesion of BSA $(0.46 \%), \operatorname{IgG}$ $(7.81 \%)$, and FN $(6.22 \%)$ was significantly reduced $(p<$ $0.001)$ on HA-coated surfaces compared to glass controls $(100 \%)$ as measured by fluorescence intensity. Typical examples of the fluorescent images for a bare silicon oxide, a HA surface after thorough washing, and an as-coated HA film are shown in Figure 3 when FN is applied to the surface with subsequent antibody staining (see the experimental protocol). As seen from the figure, HA is uniformly attached to the surface even after extensive washing. We also tested protein resistance of various other polysaccharide surfaces on glass using FN (Figure 4). Surfaces formed with other polysaccharides resisted the adsorption of FN significantly more than glass controls $(p<0.05)$. Despite this, most other polysaccharide surfaces were still significantly less resistant to FN absorption than HA coatings $(p<0.05)$.

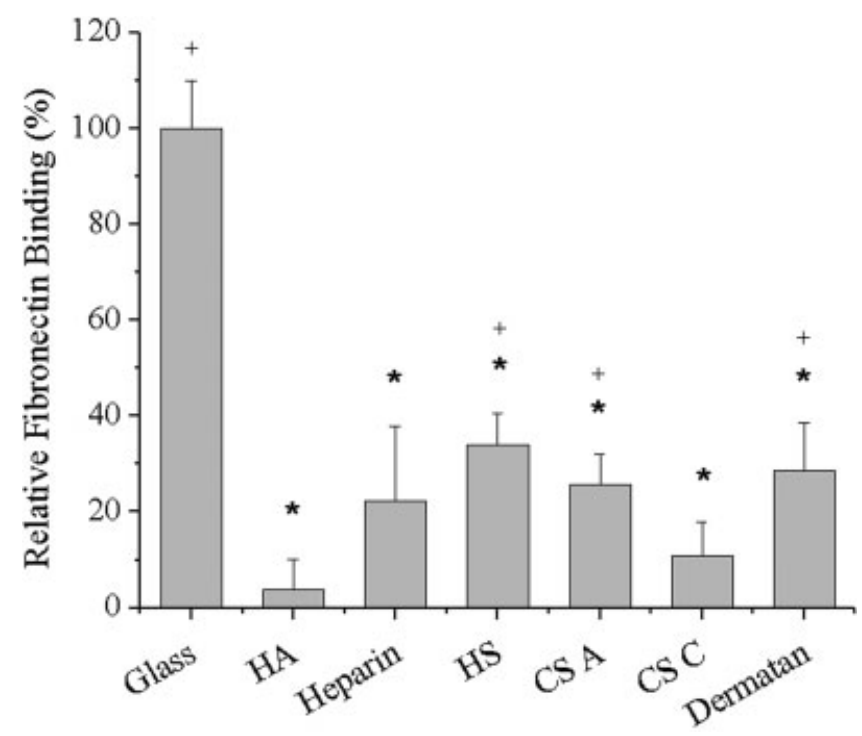

Figure 4. Fibronectin adsorption onto GAG surfaces was measured by quantifying the fluorescence intensity. The results are normalized to glass (defined as 100) as the positive control and no protein (defined as 0). Data is presented as a percentage of the difference between untreated and glass. $*$ denotes $p<0.05$ compared to glass. + denotes $p<0.05$ compared to HA. 


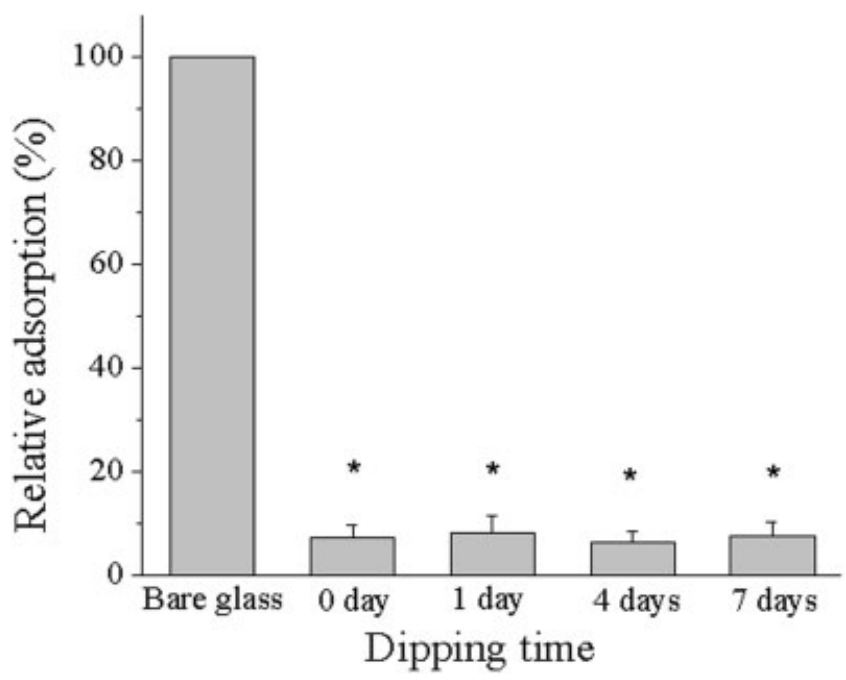

Figure 5. The stability of the HA surface was examined by the quantitative analysis of protein adsorption as a function of exposure times to PBS prior to exposure and subsequent staining to FN. Note that the surface is stable and greatly reduces protein adsorption more than $92 \%$ even after exposure to PBS for up to 7 days. No contrast enhancement was made throughout the analysis. ${ }^{*} p<0.05$.

Although HA is biodegradable in nature, the possibility of degradation can presumably be ruled out herein because oxidants such as $\mathrm{HO}$. and $\mathrm{HOCl} / \mathrm{ClO}^{-}$are believed to be important in the degradation of HA. The generation of reactive oxygen species is mediated by metal-ion catalysis $\left(\mathrm{HO}^{\circ}\right)$ in vitro. ${ }^{31,32}$ or myeloperoxidase catalyzed reaction of $\mathrm{H}_{2} \mathrm{O}_{2}$ with $\mathrm{Cl}^{-}\left(\mathrm{HOCl} / \mathrm{ClO}^{-}\right)$in vivo. To investigate long-term stability, XPS was performed on the aged samples, which revealed persistent nitrogen peaks even after a week in PBS solution. However, the uniform distribution of HA is difficult to measure by means of XPS. We therefore used fluorescent staining of the samples as a function of time to obtain a global assessment of HA adsorption. The chemisorbed HA layer was also stable for at least 7 days as determined by the analysis of fluorescent images (Figure 5). The presence of the HA surface greatly reduced the adsorption of FN (>92\%), even after the surface was exposed to PBS for 7 days prior to exposure FN adsorption and staining. These results indicate that at least in the case of silicon dioxide, the formation of a chemisorbed layer of HA is stable for at least 1 week.

\section{Summary}

Despite the water solubility and hydrophilic nature of HA, we have demonstrated that HA can be directly immobilized onto glass and silicon oxide substrates because of hydrogen bonding and high molecular weight. An ultrathin HA layer of about $3 \mathrm{~nm}$ is left behind even after extensive washing with PBS or water. The presence of this layer was verified with XPS, elliposometry, and AFM measurements. Fluorescent staining and XPS showed that the resulting surfaces remain stable for at least 7 days. Thus, our approach could be a general route to the immobilization of HA and open a new way to attach other bioactive molecules having hydrophilic moieties to solid substrates.

Dr. Suh thanks the support by the Brain Korea 21 Project in 2004 and Dr. Yang thanks the support from the National Science Council of Republic of China and the partial support from Chang Gung Hospital. D. Berry is supported by a Howard Hughes Medical Institute predoctorial fellowship. This work was supported by the U.S. Army Research Office through the Institute for Soldier Nanotechnologies of MIT (grant number: DAAD-19-02-D0002) and the content of the information does not necessarily reflect the position or policy of the Government, and no official endorsement should be inferred.

\section{REFERENCES}

1. Morra M. On the molecular basis of fouling resistance. J Biomater Sci Polym Ed 2000;11:547-569.

2. Piehler J, Brecht A, Hehl K, Gauglitz G. Protein interactions in covalently attached dextran layers. Colloids Surf B 1999;13: 325-336.

3. Osterberg E, Bergstrom K, Holmberg K, Riggs JA, Vanalstine JM, Schuman TP, Burns NL, Harris JM. Comparison of polysaccharide and poly(ethylene glycol) coatings for reduction of protein adsorption on polystyrene surfaces. Colloids Surf A 1993;77:159-169.

4. Osterberg E, Bergstrom K, Holmberg K, Schuman TP, Riggs JA, Burns NL, Van Alstine JM, Harris JM. Protein-rejecting ability of surface-bound dextran in end-on and side-on configurations: comparison to PEG. J Biomed Mater Res 1995;29: 741-747.

5. Wang D, Liu S, Trummer BJ, Deng C, Wang A. Carbohydrate microarrays for the recognition of cross-reactive molecular markers of microbes and host cells. Nat Biotechnol 2002;20: 275-281.

6. Lofas S, Johnsson B. A novel hydrogel matrix on gold surfaces in surface-plasmon resonance sensors for fast and efficient covalent immobilization of ligands. J Chem Soc Chem Commun 1990:1526-1528.

7. Dai L, Zientek P, St Johns H, Pasic P, Chatelier R, Griesser HJ. Surface modification of polymeric biomaterials. In: Ratner B, Castner D, editors. Surface modification of polymeric biomaterials. New York; Plenum Press: 1996. p 147.

8. Hartley PG, McArthur SL, McLean KM, Griesser HJ. Physicochemical properties of polysaccharide coatings based on grafted multilayer assemblies. Langmuir 2002;18:2483-2494.

9. Morra M, Cassineli C. Non-fouling properties of polysaccharide-coated surfaces. J Biomater Sci Polym Ed 1999;10:1107_ 1124.

10. Morra M, Cassinelli C, Pavesio A, Renier D. Atomic force microscopy evaluation of aqueous interfaces of immobilized hyaluronan. J Colloid Interface Sci 2003;259:236-243.

11. Yoshioka T, Tsuru K, Hayakawa S, Osaka A. Preparation of alginic acid layers on stainless-steel substrates for biomedical applications. Biomaterials 2003;24:2889-2894.

12. Bulpitt P, Aeschlimann D. New strategy for chemical modification of hyaluronic acid: preparation of functionalized derivatives and their use in the formation of novel biocompatible hydrogels. J Biomed Mater Res 1999;47:152-169.

13. Oerther S, Le Gall H, Payan E, Lapicque F, Presle N, Hubert P, Dexheimer J, Netter P. Hyaluronate-alginate gel as a novel biomaterial: mechanical properties and formation mechanism. Biotechnol Bioeng 1999;63:206-215.

14. Abantangelo G, Weigel P. New frontiers in medical science: redefining hyaluronan. Amsterdam; Elsevier: 2000.

15. Balazs EA, Denlinger JL. Clinical uses of hyaluronan: the biology of hyaluronan. In: Evered D, Welan J, editors. Clinical 
uses of hyaluronan: the biology of hyaluronan. New York; Wiley: 1989. p 265-280.

16. Piacquadio D, Jarcho M, Goltz R. Evaluation of hylan b gel as a soft-tissue augmentation implant material. J Am Acad Dermatol 1997;36:544-549.

17. Pei M, Solchaga LA, Seidel J, Zeng L, Vunjak-Novakovic G, Caplan AI, Freed LE. Bioreactors mediate the effectiveness of tissue engineering scaffolds. FASEB J 2002;16:1691-1694.

18. Bernard BA, Newton SA, Olden K. Effect of size and location of the oligosaccharide chain on protease degradation of bovine pancreatic ribonuclease. J Biol Chem 1983;258:12198-12202.

19. Lohmander LS, De Luca S, Nilsson B, Hascall VC, Caputo CB, Kimura JH, Heinegard D. Oligosaccharides on proteoglycans from the swarm rat chondrosarcoma. J Biol Chem 1980;255: 6084-6091.

20. Miyake K, Underhill CB, Lesley J, Kincade PW. Hyaluronate can function as a cell adhesion molecule and CD44 participates in hyaluronate recognition. J Exp Med 1990;172:69-75.

21. Mason M, Vercruysse KP, Kirker KR, Frisch R, Marecak DM, Prestwich GD, Pitt WG. Attachment of hyaluronic acid to polypropylene, polystyrene, and polytetrafluoroethylene. Biomaterials 2000;21:31-36.

22. Stile RA, Barber TA, Castner DG, Healy KE. Sequential robust design methodology and X-ray photoelectron spectroscopy to analyze the grafting of hyaluronic acid to glass substrates. J Biomed Mater Res 2002;61:391-398.

23. Chen G, Ito Y, Imanishi Y, Magnani A, Lamponi S, Barbucci R. Photoimmobilization of sulfated hyaluronic acid for antithrombogenicity. Bioconjug Chem 1997;8:730-734.

24. Thierry B, Winnik FM, Merhi Y, Tabrizian M. Nanocoatings onto arteries via layer-by-layer deposition: toward the in vivo repair of damaged blood vessels. J Am Chem Soc 2003;125: 7494-7495.

25. Picart C, Lavalle P, Hubert P, Cuisinier FJG, Decher G, Schaaf $\mathrm{P}$, Voegel JC. Buildup mechanism for poly(L-lysine)/hyaluronic acid films onto a solid surface. Langmuir 2001;17:74147424.

26. Sengupta K, Schilling J, Marx S, Markus F, Sackmann E. Supported membrane coupled ultra-thin layer of hyaluronic acid: viscoelastic properties of a tissue-surface mimetic system. Biophysical J 2003;84:381a-381a.

27. Shard AG, Davies MC, Tendler SJB, Bennedetti L, Purbrick MD, Paul AJ, Beamson G. X-ray photoelectron spectroscopy and time-of-flight SIMS investigations of hyaluronic acid derivatives. Langmuir 1997;13:2808-2814.

28. Shibasaki Y, Seki A, Takeishi N. Thermoanalytical study on anchoring effects of long-chain diynoic acids in thermal polymerization. Thermochimica Acta 1995;253:103-110.

29. Gribbon P, Heng BC, Hardingham TE. The molecular basis of the solution properties of hyaluronan investigated by confocal fluorescence recovery after photobleaching. Biophysical J 1999; 77:2210-2216.

30. Kobayashi Y, Okamoto A, Nishinari K. Viscoelasticity of hyaluronic-acid with different molecular-weights. Biorheology 1994;31:235-244.

31. Hawkins CL, Davies MJ. Degradation of hyaluronic acid, polyand monosaccharides and model compounds by hypochlorite: Evidence for radical intermediates and fragmentation. Free Radic Biol Med 1998;24:1396-1410.

32. Miller RA, Britigan BE. The formation and biologic significance of phagocyte-derived oxidants. J Invest Med 1995;43:3949. 